PREPARED FOR THE U.S. DEPARTMENT OF ENERGY, UNDER CONTRACT DE-AC02-76CH03073

PPPL-4005

PPPL-4005

UC-70

\author{
Kinetic Modifications to MHD \\ Phenomena in Toroidal Plasmas \\ by \\ C.Z. Cheng, N.N. Gorelenkov, \\ G.J. Kramer, and E. Fredrickson
}

September 2004

$\stackrel{N}{N}$

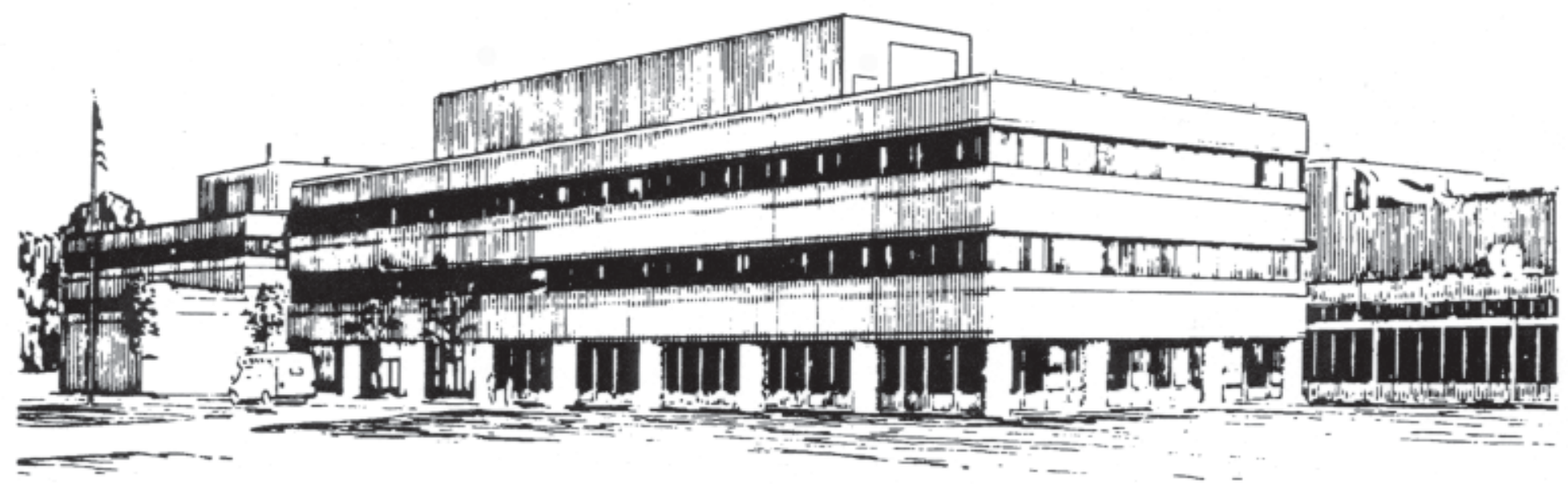

PRINCETON PLASMA PHYSICS LABORATORY PRINCETON UNIVERSITY, PRINCETON, NEW JERSEY 


\section{PPPL Reports Disclaimer}

This report was prepared as an account of work sponsored by an agency of the United States Government. Neither the United States Government nor any agency thereof, nor any of their employees, makes any warranty, express or implied, or assumes any legal liability or responsibility for the accuracy, completeness, or usefulness of any information, apparatus, product, or process disclosed, or represents that its use would not infringe privately owned rights. Reference herein to any specific commercial product, process, or service by trade name, trademark, manufacturer, or otherwise, does not necessarily constitute or imply its endorsement, recommendation, or favoring by the United States Government or any agency thereof. The views and opinions of authors expressed herein do not necessarily state or reflect those of the United States Government or any agency thereof.

\section{Availability}

This report is posted on the U.S. Department of Energy's Princeton Plasma Physics Laboratory Publications and Reports web site in Fiscal Year 2004. The home page for PPPL Reports and Publications is: http://www.pppl.gov/pub_report/

DOE and DOE Contractors can obtain copies of this report from:

U.S. Department of Energy

Office of Scientific and Technical Information

DOE Technical Information Services (DTIS)

P.O. Box 62

Oak Ridge, TN 37831

Telephone: (865) 576-8401

Fax: (865) 576-5728

Email: reports@adonis.osti.gov

This report is available to the general public from:

National Technical Information Service

U.S. Department of Commerce

5285 Port Royal Road

Springfield, VA 22161

Telephone: $1-800-553-6847$ or

(703) $605-6000$

Fax: (703) 321-8547

Internet: http://www.ntis.gov/ordering.htm 


\title{
Kinetic modifications to MHD phenomena in toroidal plasmas
}

\author{
C. Z. Cheng, N. N. Gorelenkov, G. J. Kramer and E. \\ FREDRICKSON
}

\begin{abstract}
Princeton Plasma Physics Laboratory, Princeton University, Princeton, New Jersey
\end{abstract}

\begin{abstract}
Particle kinetic effects involving small spatial and fast temporal scales can strongly affect MHD phenomena and the long time behavior of plasmas. In particular, kinetic effects such as finite ion gyroradii, trapped particle dynamics and wave-particle resonances have been shown to greatly modify the stability of MHD modes. Here, the kinetic effects of trapped electron dynamics and finite ion gyroradii are shown to have a large stabilizing effect on kinetic ballooning modes in low aspect ratio toroidal plasmas such as NSTX. We also present the analysis of TAEs destabilized by fast NBI ions in NSTX experiments and TAE stability in ITER due to $\alpha$-particles and MeV NNBI ions.
\end{abstract}

\section{Introduction}

Most studies of global phenomena are based on the MHD model. The fundamental shortcomings of the MHD model are: (a) based on the Ohm's law the plasma is frozen in the field lines and moves across the magnetic field with $\mathbf{E} \times \mathbf{B}$ drift and the parallel electric field is vanishingly small due to plasma resistivity; (b) the plasma pressure changes adiabatically according to the adiabatic pressure law; and (c) the gyro-viscous tensor that contains finite particle gyroradius effects is ignored. The Ohm's law and adiabatic pressure law are appropriate only if the frequency, $\omega$, and perturbation wavenumber, $\mathbf{k}$, satisfy the ordering assumptions that $\omega_{c i}>>>>\omega_{b}, \omega_{d}$ for both 
electrons and ions and $L>k^{-1}>>\rho_{i}$, where $\omega_{c i}$ is the ion cyclotron frequency, $\omega_{b}$ is the bounce (or transit) frequency, $\omega_{d}$ is the particle magnetic drift frequency, $L$ is the plasma and magnetic field scale length, and $\rho_{i}$ is the ion gyroradius. These assumptions can break down for many critical plasma phenomena in toroidal plasmas. For example, thermal particle kinetic effects have been shown to affect the stability of the ballooning modes $[1,2,3,4]$. Moreover, fast ion kinetic effects have been shown to destabilize the Toroidicity-Induced Alfvén Eigenmode (TAE) $[5,6,7]$ and modify the nature of internal kink modes (fishbones and sawtooth stabilization).

In this paper we present kinetic effects on the stability of kinetic ballooning modes (KBMs) and TAEs. We first show that the combined kinetic effects of trapped electron dynamics and ion finite Larmor radii (FLR) can greatly stabilize the ballooning modes. Then, we show that TAEs can be destabilized by resonant interaction with energetic ions in NSTX and ITER.

\section{Kinetic ballooning modes}

The ballooning instability which results from the release of free energy associated with nonuniform plasma pressure with a gradient in the same direction as the magnetic field curvature is one of the most serious instabilities in magnetically confined plasmas. To study the kinetic effects on ballooning modes, we consider $v_{e}>>\omega / k_{\|}>>v_{i}$, where $v_{e}$ is the electron thermal speed and $v_{i}$ is the ion thermal speed. This condition is satisfied for high temperature plasmas in most toroidal fusion devices because the frequency of KBMs is on the order of ion diamagnetic drift frequency and the temperature of electrons and ions are of the same order. Then, relative to the wave motion along the ambient magnetic field lines electrons move very rapidly with either transit or bounce motion depending on the particle pitch angle. However, ions move very slowly with respect to the parallel wave motion and their parallel dynamics can be considered as static. Moreover, electron and ion motions perpendicular to the magnetic field lines are very different if $k_{\perp} \rho_{i} \sim O(1)$; the electron perpendicular motion is essentially the combination of $\mathbf{E} \times \mathbf{B}$ and magnetic drift motion because of small gyroradii, but the ion perpendicular motion is governed by the $\mathbf{E} \times \mathbf{B}$, magnetic drifts as well as the polarization drift due to finite gyroradii. The difference in electron and ion perpendicular motions causes charge separation. In order to maintain charge quasi-neutral a parallel electric field $\left(\delta E_{\|}\right)$must be produced to accelerate (or decelerate) electrons to positions where there is excess charge. A $\delta E_{\|}$can easily accelerate (or decelerate) untrapped electrons to change its 
density distribution. However, it is relatively harder to change the trapped electron density distribution by $\delta E_{\|}$because of their rapid bounce motion along the field lines. Thus, a $\delta E_{\|}$enhanced by a factor of $1+O\left(n_{e} / n_{e u}\right)$, where $n_{e}$ and $n_{e u}$ are the total electron density and the untrapped electron density, respectively, will be produced to move the untrapped electrons to maintain charge quasi-neutrality. The $\delta E_{\|}$will then drive an enhanced parallel current which can greatly increase the stabilizing field line tension over the value expected from the MHD theory just like the high-pressured water in a hose increases the tension of the hose.

A set of kinetic eigenmode equations has been derived for KBMs from the gyrokinetic equations by considering $v_{e}>\omega / k_{\|}>>v_{i}$, but allowing full ion gyroradius effects [8]. To obtain an analytical understanding of the KBM stability, we also consider the limits: $\omega \sim \omega_{*} \gg \omega_{d}$ for both electrons and ions, where $\omega_{*}$ is the particle diamagnetic drift frequency, $k_{\perp} \rho_{i} \ll 1$, and $\beta_{i}, \beta_{e}<1$. For large aspect ratio with $\varepsilon=r / R<<1(r$ is the minor radius and $R$ is the major radius), the trapped electron density is much smaller than $n_{e u}$ and $n_{e} / n_{e u} \sim 1+O(\sqrt{\varepsilon})$, and the critical $\beta\left(\beta_{c}\right)$ for the first (second) KBM stability is larger (smaller) than the ideal MHD threshold $\beta_{c}^{M H D}$ by a factor proportional to $\sqrt{\varepsilon}[2,8]$.

In the small aspect ratio limit, $n_{\text {eu }} / n_{e} \sim O\left(B / 2 B_{\max }\right)<<1$, where $B_{\max }$ is the maximum $\mathrm{B}$ along a field line, the KBM eigenmode equation is approximately given by [8]

$$
\begin{aligned}
\mathbf{B} \cdot \nabla\left(\frac{k_{\perp}^{2}}{B^{2}} \mathbf{B} \cdot \nabla S_{c} \Phi\right) & +\frac{\omega\left(\omega-\omega_{* p i}\right)}{V_{A}^{2}} k_{\perp}^{2} \Phi \\
& +\beta\left(\frac{\mathbf{B} \times \boldsymbol{\kappa} \cdot \mathbf{k}_{\perp}}{B}\right)\left(\frac{\mathbf{B} \times \nabla P \cdot \mathbf{k}_{\perp}}{B P}\right) \Phi \simeq 0,
\end{aligned}
$$

where

$$
\begin{aligned}
S_{c}=1+\frac{n_{e}}{n_{e u}}\left[\frac{\beta_{e}}{2}\right. & \left(\frac{\omega_{* p i}-\omega_{* p e}}{\omega}\right)^{2}-\frac{q_{i} T_{e}}{q_{e} T_{i}}\left(\frac{\omega-\omega_{* p i}}{\omega-\omega_{* e}}\right) b_{i} \\
& \left.-\frac{3}{2}\left(\frac{\omega-\omega_{* p e}}{\omega-\omega_{* e}}\right) \frac{\left\langle\hat{\omega}_{d e}\right\rangle}{\omega}+\left(\frac{\omega-\omega_{* p i}}{\omega-\omega_{* e}}\right) \frac{\hat{\omega}_{B e}+\hat{\omega}_{k e}}{2 \omega}\right],
\end{aligned}
$$

$b_{i}=k_{\perp}^{2} T_{i} / m_{i} \omega_{c i}^{2}<1, \omega_{* j}=\mathbf{B} \times \nabla \ln n_{j} \cdot \mathbf{k}_{\perp} T_{j} / q_{j} B^{2}, j=i, e$ indicates the particle species, $\omega_{* p j}=\omega_{* j}\left(1+\eta_{j}\right), \eta_{j}=d \ln T_{j} / d \ln n_{j}, \hat{\omega}_{B j}=2 \mathbf{B} \times \nabla B$. $\mathbf{k}_{\perp} T_{j} / q_{j} B^{3}, \hat{\omega}_{k j}=2 \mathbf{B} \times \boldsymbol{\kappa} \cdot \mathbf{k}_{\perp} T_{j} / q_{j} B^{2}$, and $\left\langle\hat{\omega}_{d e}\right\rangle$ is the bounce-averaged electron magnetic drift frequency evaluated at its thermal energy.

Note that the coefficient of $n_{e} / n_{e u}$ in Eq. (2) is positive and thus $S_{c}>1$. For $n_{e} / n_{e u}>>1$ and $b_{i} \sim O(1)$ or $\beta \sim O(1)$, the $n_{e} / n_{e u}$ term is much 
larger than unity, and $S_{c}>>1$. Physically, $S_{c}$ is related to the perturbed parallel current, given by $\delta J_{\|} \simeq i \nabla_{\perp}^{2} \nabla_{\|}\left(S_{c} \Phi\right) / \omega$, which gives rise to an enhanced stabilizing field line tension due to an enhanced $\delta E_{\|}$resulting from the combined kinetic effects of trapped electron dynamics, ion FLR and magnetic drift motion. The local dispersion relation is given by

$$
\frac{\omega\left(\omega-\omega_{* p i}\right)}{V_{A}^{2}}+\beta\left(\frac{\mathbf{B} \times \boldsymbol{\kappa} \cdot \mathbf{k}_{\perp}}{k_{\perp} B}\right)\left(\frac{\mathbf{B} \times \nabla \ln P \cdot \mathbf{k}_{\perp}}{k_{\perp} B}\right) \simeq S_{c} k_{\|}^{2} .
$$

At marginal stability the critical $\beta$ for the KBM is given by

$$
\beta_{c} \simeq S_{c} \beta_{c}^{M H D}-\frac{\omega\left(\omega-\omega_{* p i}\right) R_{c} L_{p}}{V_{A}^{2}}
$$

where $\beta_{c}^{M H D}=k_{\|}^{2} R_{c} L_{p}$ is the ballooning instability threshold based on the ideal MHD theory, $R_{c}$ is the radius of the magnetic field curvature and $L_{p}$ is the pressure gradient scale length. For $\eta_{i}=0, \omega=\omega_{* i}$ at the marginal stability. Then, the second term on the right hand side of Eq. (4) vanishes and $\beta_{c}=S_{c} \beta_{c}^{M H D}$ is enhanced over the ideal MHD threshold by $S_{c}$. For $\eta_{i} \neq 0, \omega>\omega_{* p i}$ at the marginal stability, thus $\omega\left(\omega-\omega_{* p i}\right)>0$ and the second term on the right hand side of Eq. (4) reduces the $\beta_{c}$. However, $S_{c}$ is also modified by the $\omega$ value at the marginal stability.

For small aspect ratio with $r / R \sim O(1)$, the fraction of trapped electron population is much larger than the untrapped fraction and $n_{e u} / n_{e} \simeq 1-$ $\sqrt{1-(R-r) /(R+r)} \simeq(R-r) / 2(R+r)<<1$. Then, the first stability $\beta_{c}$ of the KBM is enhanced over $\beta_{c}^{M H D}$ by $O\left(n_{e} / n_{e u}\right)$. For an aspect ratio of $R / r=1.5$ as in NSTX, $\beta_{c}$ can be a factor of $n_{e u} / n_{e} \simeq 10$ larger than $\beta_{c}^{M H D}$ and we expect the KBM to be stable in the finite magnetic shear region. However, if NSTX has a reverse shear, then the KBM is expected to be unstable around the radial location of the minimum safety factor, where the magnetic shear is very weak.

\subsection{KBM stability in large aspect ratio $\left(\hat{s}-\alpha_{p}\right)$ equilibrium}

Numerical studies of high- $n$ ( $n$ is the toroidal mode number) KBMs based on the gyrokinetic eigenmode equations have been performed for circular cross section tokamaks $[1,2]$ by employing the $\left(\hat{s}-\alpha_{p}\right)$ analytical model equilibrium where $\hat{s}=r q^{\prime} / q$ is the magnetic shear, $\alpha_{p}=-2 P^{\prime} R q^{2} / B_{0}^{2}, q$ is the safety factor, $P$ is the plasma pressure, $B_{0}$ is the averaged magnetic field intensity over a flux surface. We choose the fixed parameters to be $b_{\theta}=k_{\theta}^{2} \rho_{i}^{2} / 2=0.1, \varepsilon_{n}=L_{n} / R=0.1$, where $L_{n}=\left(d \operatorname{lnn}_{i} / d r\right)^{-1}, \hat{s}=0.5$, 


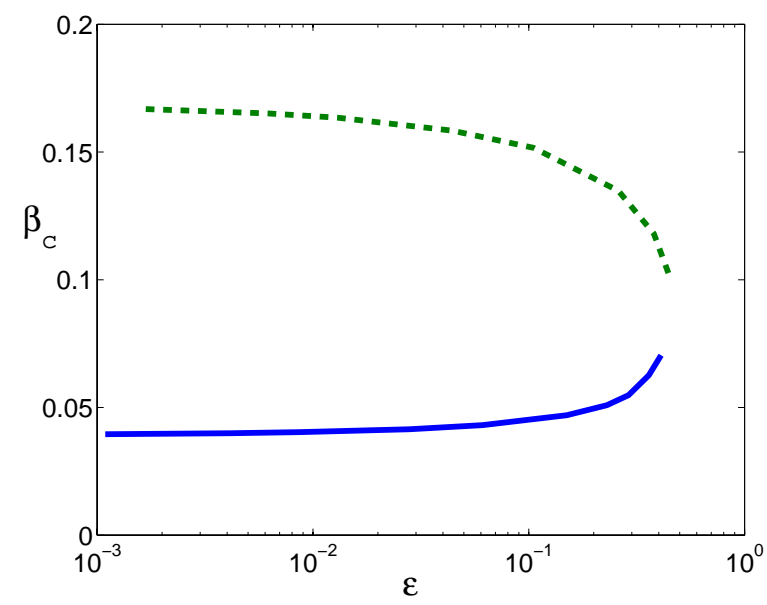

Figure 1: Critical $\beta$ of the first (dash curve) and second (solid curve) stability of the kinetic ballooning mode versus the inverse aspect ratio $\varepsilon=r / R$ for a large aspect ratio $\left(\hat{s}-\alpha_{p}\right)$ equilibrium for the fixed parameters: $b_{\theta}=0.1, \varepsilon_{n}=0.1$, $\hat{s}=0.5, q=1, \eta_{e}=\eta_{i}=0, T_{e} / T_{i}=1$. For $\beta$ values between these two curves ideal ballooning modes are unstable.

$q=T_{e} / T_{i}=1, \eta_{i}=d \ln T_{i} / d \ln n_{i}=0, \eta_{e}=d \ln T_{e} / d \ln n_{e}=0$. Then, $\omega_{* p i}=\left(1+\eta_{i}\right) \omega_{* i}$, and $\omega_{* p e}=\left(1+\eta_{e}\right) \omega_{* e}$. Figure 1 shows $\beta_{c}$ of the first and second stability boundaries for KBMs versus $\varepsilon$, which demonstrates the stabilizing effect of trapped electrons. It is clear that for small $\varepsilon$ both the increase in $\beta_{c}$ from the $\varepsilon=0$ case for the first stability boundary and the reduction in $\beta_{c}$ from the $\varepsilon=0$ case for the second stability boundary are proportional to $\sqrt{\varepsilon}$, which represents the trapped electron density fraction.

\subsection{KBM stability for NSTX equilibrium}

The stabilization kinetic effect on KBMs is more effective in small aspect ratio toroidal devices such as NSTX. To study KBMs in NSTX, we choose the baseline NSTX equilibrium parameters: $R=0.86 \mathrm{~m}$, minor radius of the last magnetic surface is $a=0.68 \mathrm{~m}(R / a=1.27)$, the last magnetic surface ellipticity is $\kappa=1.63$ and triangularity is $\delta=0.417$, the vacuum magnetic field at the geometrical axes is $B_{0}=0.3 T$. For simplicity we choose constant temperature with $T_{e}=T_{i}=1 \mathrm{keV}$ so that $\eta_{i}=\eta_{e}=0$, which eliminates the temperature gradient driven modes. The central electron density is $n_{e 0}=2.9 \times 10^{13} \mathrm{~cm}^{-3}$, the central beta is $\beta_{0} \equiv 2 P(0) / B_{0}^{2}=38 \%$, and the volume-averaged beta is $2\langle P\rangle / B_{0}^{2}=9.1 \%$. The $q$-profile is non-monotonic and has negative shear inside the $q_{\min }=q(r / a=0.3)=0.93$ surface, where 
(a)

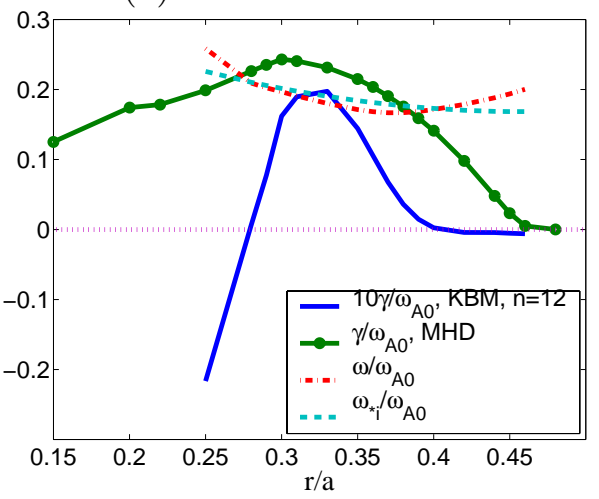

(b)

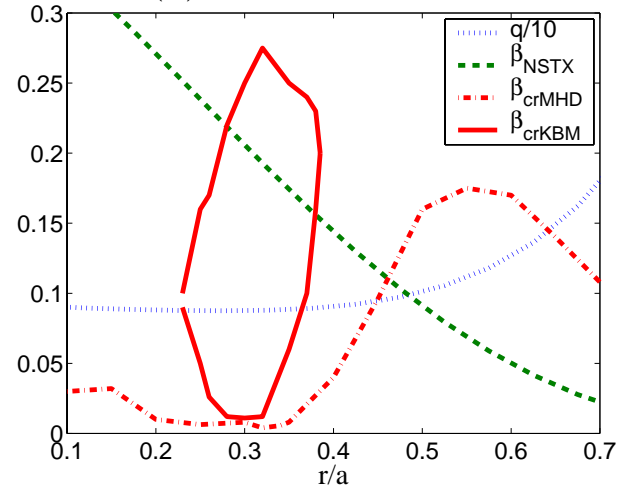

Figure 2: (a) The KBM frequency and growth rate (normalized by $\omega_{A 0}=$ $V_{A}(0) / q(0) R$ ) versus $r / a$ for $n=12$ for the baseline NSTX equilibrium. Also shown are the MHD ballooning mode growth rate and ion diamagnetic drift frequency. (b) The critical beta for the $n=12 \mathrm{KBM}$ and the ideal MHD ballooning mode for the baseline NSTX equilibria with aspect ratio $R / a=1.27$. Also shown are the NSTX $\beta$ and q profiles. The KBM is unstable for $\beta$ inside the $\beta_{\operatorname{cr} K B M}$ closed curve and the ideal ballooning modes are unstable for $\beta>\beta_{\operatorname{cr} M H D}$.

$r^{2} / a^{2} \equiv \Psi_{t o r}$, and $\Psi_{t o r}$ is the toroidal magnetic flux normalized to zero at the magnetic axis and unity at the plasma edge.

The high- $n$ gyrokinetic eigenmode equations are solved by employing the HINST code [9]. Figure 2(a) shows the dependence of the KBM growth rate and real frequency (normalized by $\left.\omega_{A 0}=V_{A}(0) / q(0) R\right)$ on $r / a$ for the baseline NSTX equilibrium for $n=12$. The KBM is unstable in the range of $0.28<r / a<0.4$, which is much smaller than the unstable region $(r / a<0.45)$ of the ideal MHD ballooning mode, whose growth rates are also shown in Fig. 2(a). Note that at marginal stability the KBM real frequency equals the ion diamagnetic drift frequency $\omega_{* i}$, consistent with our previous studies $[1,2]$. The KBM growth rate is much smaller than the MHD growth rate. Also, when the KBM is unstable, its real frequency is smaller than the local $\omega_{* i}$, and the most unstable KBM occurs near $r / a=0.33$.

Figure 2(b) shows the marginal stability boundary for KBMs and ideal MHD ballooning modes in the local $\beta$ and $r / a$ plane for $n=12$. To compute $\beta_{c}$ for each flux surface, we construct new equilibria by varying $n_{e}(0)$ (or $\beta(0)$ while keeping other equilibrium quantities and profiles fixed. For the baseline NSTX equilibrium the plasma $\beta$ is larger than $\beta_{c}$ for unstable ideal MHD ballooning modes in a very broad region $(r / a<0.46)$ as shown in Fig. 2(b). However, the stability boundary for KBMs forms a closed curve and KBMs are stable outside this closed curve. For the NSTX baseline 
equilibrium KBMs are unstable from $r / a=0.28$ to $r / a=0.37$ with the most unstable region near the zero shear surface at $r / a \simeq 0.3$. Note that the HINST calculations do not find unstable KBMs up to $\beta(0)=1$. Because the average $\beta$ for the NSTX equilibrium is $\langle\beta\rangle=9.1 \%$ and the experimentally achieved values are about a factor of three larger, we expect full stabilization of KBMs in high $\beta$ NSTX discharges.

\section{TAE instabilities}

TAEs can be destabilized by resonant interaction with energetic ions. The TAE frequency is $\omega \sim V_{A} / 2 q R$, where $V_{A}$ is the Alfvén velocity. TAEs can resonate with energetic particles with velocity $V_{h} \sim V_{A}$ and are destabilized by the energetic particle phase space gradient (pressure gradient and positive energy gradient). For slowing-down energy distribution, fast particles can drive TAEs when $n q\left(V_{h} / V_{A}\right) \geq\left(r L_{h} / R \rho_{h}\right)$, where $\rho_{h}$ and $L_{h}$ are the hot ion gyroradius and pressure gradient scale lengths, respectively. Theories and experiments have confirmed that large amplitude TAEs can lead to expulsion of fast ions, degrade ignition margin and produce localized heating or damage on plasma facing components. In burning plasmas TAEs can be destabilized by $3.5 \mathrm{MeV} \alpha$-particles and can cause premature loss of $\alpha$ particles from the confinement system. However, TAEs can be unstable only if the fast particle drive overcomes bulk plasma damping effects of electron and ion Landau damping, radiation and continuum damping.

We will address the TAE stability in NSTX and in the proposed ITER experiments. For NSTX we present TAE experimental results and compare them with the theoretical analysis. For ITER we present TAE stability analysis and summarize the parameter domain where TAEs are expected to be unstable due to $\alpha$-particles and MeV NNBI ions. The stability of TAEtype modes is analyzed by employing global kinetic-MHD stability codes, NOVA/NOVA-K codes $[10,11,12]$.

\subsection{TAEs in NSTX}

NSTX is an excellent device for studying TAE stability relevant to burning plasmas because $1<v_{b} / V_{A}<3$ in NSTX (in ITER $1<v_{\alpha} / V_{A}<2$, where $v_{\alpha}$ is the alpha birth velocity). TAE modes have been observed in NSTX even for modest NBI power [13]. Because of low aspect ratio the toroidal coupling effect is strong. The Alfvén continuum gap is wide open across the minor radius and a broad spectrum of TAEs can exist [14] for each toroidal

mode number $n$. Figure 3(a) shows a broad spectrum of TAEs observed 

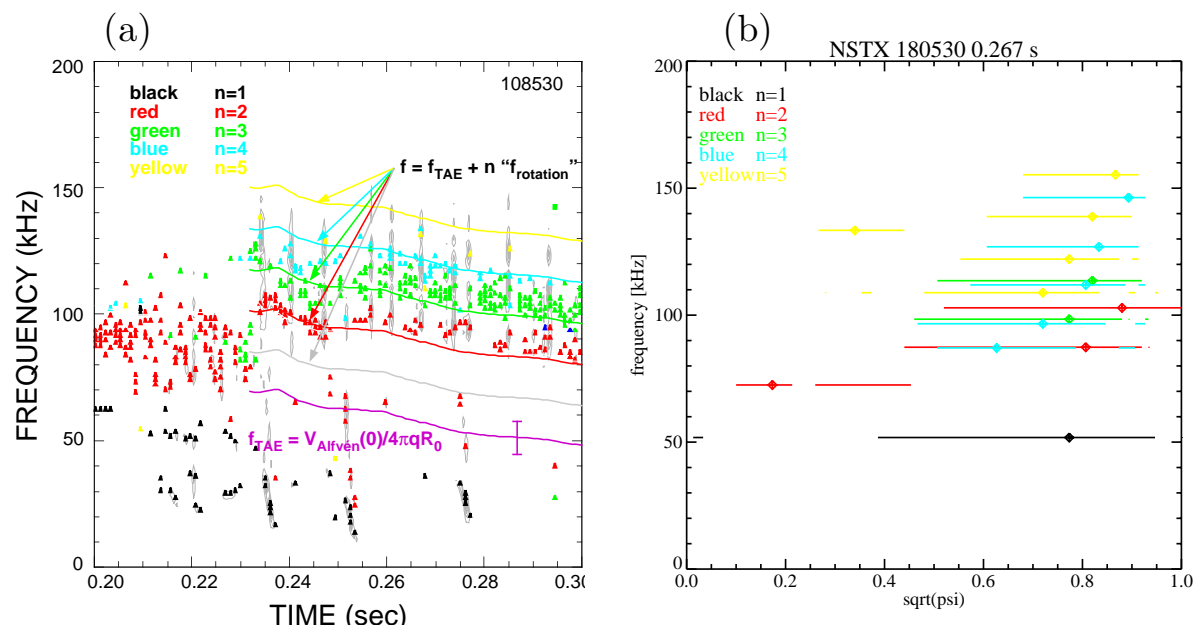

Figure 3: (a) TAE spectrum observed in the NSTX shot 108530. (b) TAE spectrum computed by the NOVA/NOVA-K codes for the NSTX shot 108530 at 0.267 sec.

with several frequencies for each $n$ for the NSTX shot 108530. The plasma parameters for this shot are $B_{0}=0.434 T, R=87 \mathrm{~cm}, a=63 \mathrm{~cm}$, ellipticity $\epsilon=1.74$, triangularity $\delta=0.5$, and the deuterium neutral beam is injected co-tangentially to the plasma current (but counter to the toroidal field) with an injection energy of $80 \mathrm{keV}$ and total NBI power of $3.2 \mathrm{MW}$ for two beam lines. TAEs are most commonly present in the early phase of the discharge, during the current ramp (with one NBI beam) and when the density is low $\left(2-3 \times 10^{13} \mathrm{~cm}^{-3}\right.$ on axis). A variety of modes in the frequency range from 20 to $150 \mathrm{kHz}$ with toroidal mode numbers from $n=1$ to $n=6$ are commonly observed in beam heated discharges. Higher $n$ modes are generally associated with higher frequencies, possibly related to increased Doppler shift due to toroidal plasma rotation. However, the mode spacing is not nearly as uniform. In addition to the more commonly observed quasicontinuous TAEs, bursting TAEs (indicated by vertical dashed lines) are also observed after the second beam is injected at $0.21 \mathrm{sec}$. These bursting modes are associated with fast neutron drops, $H_{\alpha}$ micro-bursts, and $5-10 \%$ fast ions hitting the wall. Each burst consists of multiple modes with $n$ ranging 2 - 5 with a dominant mode being $n=2$ or 3 . From the bursting mode amplitude modulation one sees beating of multiple modes, but the bursting fluctuation is dominated by a single frequency mode with mode growth and decay times approximately $50-100 \mu s$.

To understand the observed TAE spectrum we perform the NOVA/NOVA-K calculation for the NSTX discharge at $0.267 \mathrm{sec}$ and the 
results are shown in Figure 3(b), which shows a very rich TAE frequency spectrum of unstable TAE modes and the radial extent of TAEs for $n=1-5$ modes. In Fig. 3(b) the dotted mark on each horizontal bar indicates the radial location of the maximum amplitude of the TAE poloidal harmonics and the bar shows the radial width that the amplitude of the TAE poloidal harmonics is at least one-half of the maximum amplitude. Note that for each $n$ there are multiple TAEs. To compute the TAE spectrum for the NSTX discharge, the NSTX equilibrium is constructed by ignoring the toroidal rotation effect and choosing the plasma profiles modeled by the TRANSP code, $q_{a}=11.4, n_{e}(0)=2.54 \times 10^{13} \mathrm{~cm}^{3}, \beta(0)=21.4 \%$, $\langle\beta\rangle \sim 2.88 \%$. Because the plasma toroidal rotation velocity is significant with $V_{\text {rot }}(0) \simeq 170 \mathrm{~km} / \mathrm{s}$ at $0.27 \mathrm{sec}$ and has a peak profile with half width at $r / a=0.5$, the frequencies shown in Fig. 3(b) are those computed by the NOVA code that uses the Doppler-shifted frequency $f=f_{T A E}+n f_{\text {rot }}(r)$, where $f_{\text {rot }}(r)=V_{\text {rot }}(r) / 2 \pi R$ is the radial dependence of the toroidal rotation frequency, $V_{\text {rot }}$ is the toroidal rotation velocity versus $r / a=\sqrt{\Psi}$, and $\Psi$ is the poloidal flux normalized to zero at the magnetic axis and unity at the plasma edge. For $V_{\text {rot }}=100 \mathrm{~km} / \mathrm{s}$ and $R=1 \mathrm{~m}$, we have $f_{\text {rot }} \simeq 16 \mathrm{kHz}$.

From Fig. 3 we compare the TAE frequencies obtained from the NOVA calculations with the NSTX observations at $0.267 \mathrm{sec}$. Note that from the NOVA calculations the $n=1$ mode frequency is about $50 \mathrm{kHz}$ which is about $15 k \mathrm{~Hz}$ higher than the NSTX result. For $n=2$ there are 3 unstable TAEs with frequencies being $72 \mathrm{kHz}, 88 \mathrm{kHz}$, and $102 \mathrm{kHz}$ which are consistent with the observed frequency range of $90-100 k H z$. For $n=3$ there are 2 unstable TAEs with frequencies being $99 k \mathrm{~Hz}$ and $103 \mathrm{kHz}$ which are consistent with the observed frequency range of $102-120 k H z$. For $n=4$ there are 5 unstable TAEs with frequencies ranging from $88 \mathrm{kHz}$ to $147 \mathrm{kHz}$, consistent with the observed frequency range of $110-135 \mathrm{kHz}$ but not as good as the other $n$ cases. For $n=5$ there are 5 unstable TAEs with frequencies ranging from $110 \mathrm{kHz}$ to $155 \mathrm{kHz}$ which are also consistent with the observed frequency range of $115-140 \mathrm{kHz}$. Thus, considering the uncertainty in the measurement of the plasma profiles and the effect of plasma rotation, the theoretical results are reasonably consistent with observations.

The growth rates of these TAEs are computed by feeding these Dopplershifted frequencies into the NOVA-K code. To model the beam ion distribution we assume a slowing down distribution in energy, multiplied by a weighting factor $f_{\lambda}(\lambda)=\left(4 / \Delta_{\lambda} \sqrt{\pi}\right) e^{-\lambda^{2} / \Delta_{\lambda}^{2}}$, where $\lambda=v_{\perp}^{2} / B v^{2}$ and $\Delta_{\lambda}$ is the pitch angle width and is chosen to be from 0.25 to 0.5 . These $n=1-5$ TAEs with peak amplitude located around $\sqrt{\Psi}<0.9$ are destabilized by the NBI ions. The calculated linear growth rates are about one percent of 
(a)

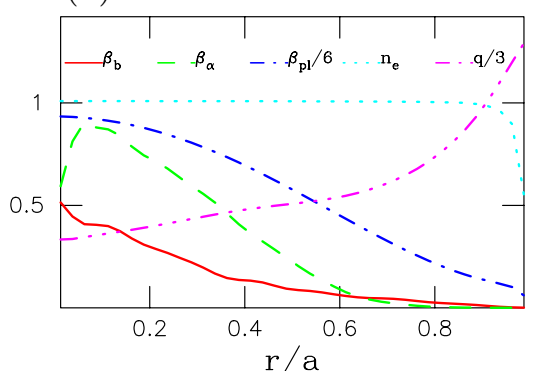

(b)

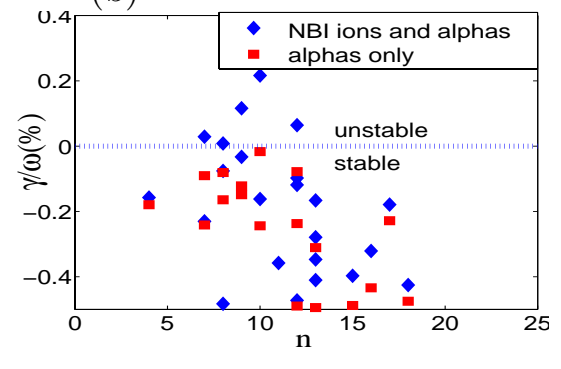

Figure 4: (a) Profiles of the beam beta $\beta_{b}$ (in $\%$ ), the fusion alpha beta $\beta_{\alpha}$ (in $\%$ ), safety factor $q$ and the electron density $n_{e}$ (in $10^{14} \mathrm{~cm}^{-3}$ ) for the ITER plasma equilibrium as computed by TRANSP code. (b) The ratio of TAE growth rate over real frequency $(\gamma / \omega)$ versus the toroidal mode number with $\alpha$-particle drive only (squares), and with both NBI ion and $\alpha$-particle drives (diamonds).

the real frequency for $\beta_{h}(0) \simeq 13 \%$.

\subsection{TAEs in ITER}

The TAE stability in ITER has been studied by employing the perturbative NOVA-K code [15]. The equilibrium plasma profiles of the ITER nominal discharge are calculated by the transport code TRANSP and are shown in Fig. 4(a). Other plasma parameters are: $R=6.2 m, a=2 m, B_{0}=5 T$, $T_{i 0}=19.5 \mathrm{keV}, T_{e 0}=23.5 \mathrm{keV}$, and $\beta_{0}=6.7 \%$. The deuterium negatively charged neutral beam injection (NNBI) power is $P_{N B I}=33 \mathrm{MW}$ at $1 \mathrm{MeV}$

injection energy and is applied tangentially aiming at the magnetic axis so that the beam ion $\beta$ peaks at the center. Also note that by choosing a flat electron density profile for this ITER equilibrium TAEs will be less unstable than for equilibria with a more peak density profile.

The dependence of $\gamma / \omega$ ( $\gamma$ is the growth rate) on the toroidal mode number for TAEs is shown in Fig. 4(b). Note that for each $n$ there are more than one TAE and most TAEs are stable. The range of unstable $n$-values spans from 7 to 12 when both $\alpha$-particle and beam ion drives are included. If only the $\alpha$-particle drive is included, TAEs are near marginal stability. As a measure of how close TAEs are to the marginal stability we note that the growth rate from alphas plus beam ions for $n=10$ is $\sim 1.5 \%$ of the real frequency. However, when the damping effect from thermal particles is included, $\gamma / \omega$ is reduced to $0.2 \%$. The damping is strong at the plasma center due to the ion Landau damping and at the plasma edge due to the trapped electron collisional damping.

The calculations also show that the more the beam is directed off-axis 
vertically the stronger the beam ion drive is. This is because during the on-axis beam injection, the beam ion beta builds up near the plasma center, where the ion Landau damping is very strong. With the off-axis beam injection the region of strong beam ion beta gradient shifts outward to the middle of the minor radius, where the TAE modes are located. Thus, for the off-axis beam injection at $0.55 \mathrm{~m}$ the instability drive increases to $\gamma / \omega=$ $0.4 \%$ (from $0.2 \%$ for the on-axis beam injection case). For the higher ion temperature case, $T_{i 0}=25.3 \mathrm{keV}$, the $\alpha$-particle destabilization of the TAE is enhanced due to higher $\beta_{\alpha 0} \simeq 1.33 \%$. However, the $n=10$ TAE is still marginally stable if only the $\alpha$-particle drive is included. However, including the beam ion drive the total instability drive is strong enough to destabilize TAEs with $\gamma / \omega$ increased to $0.55 \%$ for the most unstable mode. Finally, the contribution to the TAE drive from alphas or beam ions is much smaller than the TAE real frequency (typically at $\sim 1-3 \%$ ), and thus the perturbative approach employed by the NOVA-K code should be adequate.

\section{Summary}

In summary, we have presented kinetic effects on MHD type modes by considering (1) the stabilization of KBMs by the combined effect of trapped electron dynamics and ion FLR, and (2) the destabilization of TAEs in NSTX by fast NBI ions and in ITER by $\alpha$-particles and MeV NNBI ions.

It is difficult to model the kinetic modifications to MHD phenomena mainly because of the disparate scales between kinetic physics and MHD phenomena: global scale MHD phenomena are generally studied using the MHD framework, while microscale kinetic phenomena are described with kinetic theories. To accommodate the advantages of both MHD and kinetic models, we have developed a nonlinear kinetic-fluid model for modeling kinetic effects to MHD phenomena [16]. The model consists of single-fluid equations coupled with gyrokinetic or full Vlasov descriptions for all particle species via pressure tensors. In the linear limit, we have shown that the kinetic-fluid model properly retains key physics of both thermal and energetic particles for global MHD modes. The kinetic-fluid model is a natural extension of the previously developed kinetic-MHD model [17], which has been successfully employed to build global linear and nonlinear kinetic-MHD codes $[11,18]$ for modeling fast ion driven modes.

Acknowledgments: This work is supported by US DOE contract No. DE-AC02-76CH03073. 


\section{References}

[1] CHENG C. Z., Phys. Fluids, 25 (1982) 1020.

[2] CHENG C. Z., Nucl. Fusion, 22 (1982) 773.

[3] TANG W. M. ET AL., Nucl. Fusion, 25 (1985) 151.

[4] HAStiE R. J., CATto P. J. AND RAMOs J. J., Phys. Plasmas, 7 (2000) 4561.

[5] Cheng, C. Z., CHEN L. AND CHAnCE M. S., Ann. Phys.(NY), 161 (1985) 21.

[6] CHENG C. Z. AND CHANCE M. S., Phys. Fluids, 29 (1986) 3695.

[7] CHENG C. Z., GORELENKOV N. N. AND HSU C. T., Nucl. Fusion, 35 (1995) 1639.

[8] CHEng C. Z. AND GORElEnKOV N. N., Phys. Plasmas, in press (2004).

[9] Gorelenkov N. N., CHEnG C. Z. AND TANG W., Phys. Plasmas, 5 (1998) 3389.

[10] CHEng C. Z. AND CHAnCE M. S., J. Comput. Phys., 71 (1987) 124.

[11] CHENG C. Z., Phys. Reports, 211 (1992) 1.

[12] GORElenkov N. N., CHEnG C. Z. AND FU G. Y., Phys. Plasmas, 6 (1999) 2802.

[13] FREDRICKSON E.D. ET AL., Phys. Plasmas, 10 (2003) 2852.

[14] GORELEnKOV N. N. ET AL., Phys. Plasmas, 7 (2000) 1433.

[15] GORELENKOV N. N. ET AL., Nucl. Fusion, 43, (2003) 594.

[16] CHENG, C. Z. AND JOHNSON J. R., J. Geophys. Res., 104 (1999) 413.

[17] CHEnG, C. Z., J. Geophys. Res., 96 (1991) 21159.

[18] PARK, W. ET AL.,Phys. Fluids B, 4 (1992) 2033. 


\section{External Distribution}

Plasma Research Laboratory, Australian National University, Australia

Professor I.R. Jones, Flinders University, Australia

Professor João Canalle, Instituto de Fisica DEQ/IF - UERJ, Brazil

Mr. Gerson O. Ludwig, Instituto Nacional de Pesquisas, Brazil

Dr. P.H. Sakanaka, Instituto Fisica, Brazil

The Librarian, Culham Laboratory, England

Mrs. S.A. Hutchinson, JET Library, England

Professor M.N. Bussac, Ecole Polytechnique, France

Librarian, Max-Planck-Institut für Plasmaphysik, Germany

Jolan Moldvai, Reports Library, Hungarian Academy of Sciences, Central Research Institute for Physics, Hungary

Dr. P. Kaw, Institute for Plasma Research, India

Ms. P.J. Pathak, Librarian, Institute for Plasma Research, India

Ms. Clelia De Palo, Associazione EURATOM-ENEA, Italy

Dr. G. Grosso, Instituto di Fisica del Plasma, Italy

Librarian, Naka Fusion Research Establishment, JAERI, Japan

Library, Laboratory for Complex Energy Processes, Institute for Advanced Study, Kyoto University, Japan

Research Information Center, National Institute for Fusion Science, Japan

Dr. O. Mitarai, Kyushu Tokai University, Japan

Dr. Jiangang Li, Institute of Plasma Physics, Chinese Academy of Sciences, People's Republic of China

Professor Yuping Huo, School of Physical Science and Technology, People's Republic of China

Library, Academia Sinica, Institute of Plasma Physics, People's Republic of China

Librarian, Institute of Physics, Chinese Academy of Sciences, People's Republic of China

Dr. S. Mirnov, TRINITI, Troitsk, Russian Federation, Russia

Dr. V.S. Strelkov, Kurchatov Institute, Russian Federation, Russia

Professor Peter Lukac, Katedra Fyziky Plazmy MFF UK, Mlynska dolina F-2, Komenskeho Univerzita, SK-842 15 Bratislava, Slovakia

Dr. G.S. Lee, Korea Basic Science Institute, South Korea

Institute for Plasma Research, University of Maryland, USA

Librarian, Fusion Energy Division, Oak Ridge National Laboratory, USA

Librarian, Institute of Fusion Studies, University of Texas, USA

Librarian, Magnetic Fusion Program, Lawrence Livermore National Laboratory, USA

Library, General Atomics, USA

Plasma Physics Group, Fusion Energy Research Program, University of California at San Diego, USA

Plasma Physics Library, Columbia University, USA

Alkesh Punjabi, Center for Fusion Research and Training, Hampton University, USA

Dr. W.M. Stacey, Fusion Research Center, Georgia Institute of Technology, USA

Dr. John Willis, U.S. Department of Energy, Office of Fusion Energy Sciences, USA

Mr. Paul H. Wright, Indianapolis, Indiana, USA 
The Princeton Plasma Physics Laboratory is operated by Princeton University under contract with the U.S. Department of Energy.

\author{
Information Services \\ Princeton Plasma Physics Laboratory \\ P.O. Box 451 \\ Princeton, NJ 08543
}

Phone: 609-243-2750

Fax: 609-243-2751

e-mail: pppl_info@pppl.gov

Internet Address: http://www.pppl.gov 